\title{
LINEAR OPERATORS ON NON-LOCALLY CONVEX ORLICZ SPACES
}

\author{
MARIAN NOWAK and AGNIESZKA OELKE \\ Faculty of Mathematics, Computer Science and Econometrics, University of Zielona Góra \\ Szafrana 4a, 65-516 Zielona Góra, Poland \\ E-mail:M.Nowak@wmie.uz.zgora.pl,A.Oelke@wmie.uz.zgora.pl
}

\begin{abstract}
We study linear operators from a non-locally convex Orlicz space $L^{\Phi}$ to a Banach space $\left(X,\|\cdot\|_{X}\right)$. Recall that a linear operator $T: L^{\Phi} \rightarrow X$ is said to be $\sigma$-smooth whenever $u_{n} \stackrel{(\text { o) }}{\longrightarrow} 0$ in $L^{\Phi}$ implies $\left\|T\left(u_{n}\right)\right\|_{X} \rightarrow 0$. It is shown that every $\sigma$-smooth operator $T: L^{\Phi} \rightarrow X$ factors through the inclusion map $j: L^{\Phi} \rightarrow L^{\bar{\Phi}}$, where $\bar{\Phi}$ denotes the convex minorant of $\Phi$. We obtain the Bochner integral representation of $\sigma$-smooth operators $T: L^{\Phi} \rightarrow X$. This extends some earlier results of J. J. Uhl concerning the Bochner integral representation of linear operators defined on a locally convex Orlicz space.
\end{abstract}

1. Introduction and preliminaries. The theory of linear operators on Banach function spaces (in particular, $L^{p}$-spaces and Orlicz spaces $L^{\Phi}$ ) has been developed by many authors (see [D], [G], [DP], [Ph], [Z], [DS], [D $]$, [D $]$, [D $]$, [U], [C], [W]). Linear operators on non-locally convex Orlicz spaces $L^{\Phi}$ have been studied in $[\mathrm{P}],\left[\mathrm{T}_{1}\right],\left[\mathrm{T}_{2}\right],[\mathrm{K}]$.

We denote by $\sigma(L, K)$ and $\tau(L, K)$ the weak topology and the Mackey topology on $L$ with respect to the dual pair $(L, K)$. Given a topological vector space $(L, \tau)$ we will denote by $(L, \tau)^{*}$ its topological dual. For terminology concerning vector lattices and function spaces we refer to $[\mathrm{AB}],[\mathrm{KA}],[\mathrm{Z}]$.

Let $(\Omega, \Sigma, \mu)$ be a $\sigma$-finite atomless measure space, and let $L^{0}$ denote the set of $\mu$ equivalence classes of real valued measurable functions defined on $\Omega$. Then $L^{0}$ is a super Dedekind complete Riesz space under the ordering $u \leq v$ whenever $u(\omega) \leq v(\omega) \mu$-a.e. on $\Omega$. By $\mathcal{S}(\Sigma)$ we will denote the set of all $\Sigma$-simple functions defined on $\Omega$.

Now we recall notation and some basic results concerning Orlicz spaces (see $\left[\mathrm{MO}_{1}\right]$, $[\mathrm{MaO}],[\mathrm{M}],[\mathrm{RR}])$. By an Orlicz function we mean here a mapping $\Phi:[0, \infty) \rightarrow$ $[0, \infty)$ that is non-decreasing, left continuous, continuous at 0 , vanishing only at 0 and

2000 Mathematics Subject Classification: 46E30, 47B38.

Key words and phrases: Orlicz spaces, Lebesgue topology, modular topology, $\sigma$-smooth operators, Bochner integral representation, Radon-Nikodym property.

The paper is in final form and no version of it will be published elsewhere. 
$\liminf _{t \rightarrow \infty} \frac{\Phi(t)}{t}>0$. By $\Phi^{*}$ we denote the convex Orlicz function complementary to $\Phi$ in the sense of Young, i.e., $\Phi^{*}(s)=\sup \{s t-\Phi(t): t \geq 0\}$ for $s \geq 0$. Note that $\Phi^{*}$ takes only finite values whenever $\liminf _{t \rightarrow \infty} \frac{\Phi(t)}{t}=\infty$ and jumps to $\infty$ whenever $\liminf _{t \rightarrow \infty} \frac{\Phi(t)}{t}<\infty$ (see [N $\mathrm{N}_{3}$, Lemmas 2.2 and 2.3]). The function $\bar{\Phi}(t)=\left(\Phi^{*}\right)^{*}(t)$ for $t \geq 0$ is called the convex minorant of $\Phi$, because it is the largest convex Orlicz function smaller than $\Phi$ on $[0, \infty)$. Recall that $\Phi$ satisfies the $\Delta_{2}$-condition (in symb. $\Phi \in \Delta_{2}$ ) if $\Phi(2 t) \leq c \Phi(t)$ for all $t \geq 0$ and some $c>0$. An Orlicz function $\Phi$ determines a functional $\varrho_{\Phi}: L^{0} \rightarrow[0, \infty]$ by

$$
\varrho_{\Phi}(u)=\int_{\Omega} \Phi(|u(\omega)|) d \mu
$$

The Orlicz space $L^{\Phi}$ is an ideal of $L^{0}$ defined by

$$
L^{\Phi}=\left\{u \in L^{0}: \varrho_{\Phi}(\alpha u)<\infty \quad \text { for some } \alpha>0\right\}
$$

and equipped with the complete topology $\mathcal{T}_{\Phi}$ of the $F$-Riesz norm

$$
|u|_{\Phi}:=\inf \left\{\alpha>0: \varrho_{\Phi}(u / \alpha) \leq \alpha\right\}
$$

The space $\left(L^{\Phi}, \mathcal{T}_{\Phi}\right)$ is locally convex if and only if $L^{\Phi}=L^{\Phi_{0}}$ for some convex Orlicz function $\Phi_{0}$ (see [MaO]). In case $\Phi$ is a convex Orlicz function $\mathcal{T}_{\Phi}$ can be generated by two Riesz norms:

$$
\|u\|_{\Phi}:=\inf \left\{\alpha>0: \varrho_{\Phi}(u / \alpha) \leq 1\right\}
$$

and

$$
\|u\|_{\Phi}^{0}:=\sup \left\{\int_{\Omega}|u(\omega) v(\omega)| d \mu: v \in L^{\Phi^{*}}, \varrho_{\Phi^{*}}(u) \leq 1\right\} .
$$

Let $\left(L^{\Phi}\right)^{\prime}$ stand for the Köthe dual of $L^{\Phi}$. Then $\left(L^{\Phi}\right)^{\prime}=L^{\Phi^{*}}$ (see $\left[\mathrm{N}_{3}\right.$, Theorem 3.3], [MW]). Let $\left(L^{\Phi}\right)_{n}^{\sim}$ denote the order continuous dual of $L^{\Phi}$. Then $\left(L^{\Phi}\right)_{n}^{\sim}$ can be identified with $L^{\Phi^{*}}$ through the mapping: $L^{\Phi^{*}} \ni v \mapsto \varphi_{v} \in\left(L^{\Phi}\right)_{n}^{\sim}$, where

$$
\varphi_{v}(u)=\int_{\Omega} u(\omega) v(\omega) d \mu \quad \text { for all } u \in L^{\Phi} .
$$

The functional $\varrho_{\Phi}$ restricted to $L^{\Phi}$ is a modular (see $\left.\left[\mathrm{MO}_{1}\right],\left[\mathrm{MO}_{2}\right],[\mathrm{M}]\right)$. Recall that a sequence $\left(u_{n}\right)$ in $L^{\Phi}$ is said to be modularly convergent to $u \in L^{\Phi}$ (in symb. $u_{n} \stackrel{\varrho_{\Phi}}{\longrightarrow} u$ ) if $\varrho_{\Phi}\left(\alpha\left(u_{n}-u\right)\right) \rightarrow 0$ for some $\alpha>0$.

For $\varepsilon>0$ let $U_{\Phi}(\varepsilon)=\left\{u \in L^{\Phi}: \varrho_{\Phi}(u) \leq \varepsilon\right\}$. Then the family of all sets of the form: $\bigcup_{n=1}^{\infty}\left(\sum_{i=1}^{n} U_{\Phi}\left(\varepsilon_{i}\right)\right)$, where $\left(\varepsilon_{i}\right)$ is a sequence of positive numbers, forms a local base at 0 (consisting of solid subsets of $L^{\Phi}$ ) for a topology $\mathcal{T}_{\Phi}^{\wedge}$ on $L^{\Phi}$, and called the modular topology (see $\left[\mathrm{N}_{1}\right],\left[\mathrm{N}_{2}\right],\left[\mathrm{N}_{4}\right]$ ). The basic properties of $\mathcal{T}_{\Phi}^{\wedge}$ are included in the following theorem (see [ $\mathrm{N}_{1}$, Theorem 1.1], [ $\mathrm{N}_{2}$, Theorem 2.5 and 3.2], [ $\mathrm{N}_{4}$, Theorem 2.2]).

THEOREM 1.1. Let $\Phi$ be an Orlicz function. Then the following statements hold:

(i) $\mathcal{T}_{\Phi}^{\wedge}$ is the finest of all linear topologies $\xi$ on $L^{\Phi}$ for which $u_{n} \stackrel{\varrho_{\Phi}}{\longrightarrow} 0$ implies $u_{n} \stackrel{\xi}{\rightarrow} 0$.

(ii) $\mathcal{T}_{\Phi}^{\wedge}$ is the finest Lebesgue topology on $L^{\Phi}$.

(iii) $\mathcal{T}_{\Phi}^{\wedge} \subset \mathcal{T}_{\Phi}$, with equality if and only if $\Phi \in \Delta_{2}$.

(iv) $\left(L^{\Phi}, \mathcal{T}_{\Phi}^{\wedge}\right)^{*}=\left(L^{\Phi}\right)_{n}^{\sim}=\left\{\varphi_{v}: v \in L^{\Phi^{*}}\right\}$. 
(v) $\tau\left(L^{\Phi}, L^{\Phi^{*}}\right)$ is equal to the restriction of the modular topology $\mathcal{T}_{\bar{\Phi}}^{\wedge}$ i.e., $\tau\left(L^{\Phi}, L^{\Phi^{*}}\right)$ $=\mathcal{T}_{\bar{\Phi}} \Gamma_{L^{\Phi}}$. In particular, $\tau\left(L^{\Phi}, L^{\Phi^{*}}\right)=\mathcal{T}_{\Phi}^{\wedge}$ whenever $\Phi$ is convex.

In view of $[\mathrm{O}]$ the dual space $\left(L^{\Phi}\right)^{*}\left(=\left(L^{\Phi}, \mathcal{T}_{\Phi}\right)^{*}\right)$ is a Banach space under the norm

$$
\|\varphi\|_{\Phi}=\sup \left\{|\varphi(u)|: u \in L^{\Phi}, \varrho_{\Phi}(u) \leq 1\right\}
$$

for $\varphi \in\left(L^{\Phi}\right)^{*}$. Moreover, by $[\mathrm{O}, 1.31]$ the following inequality holds:

$$
|\varphi(u)| \leq\|\varphi\|_{\Phi}\left(\varrho_{\Phi}(u)+1\right) \quad \text { for all } u \in L^{\Phi} .
$$

From now on we assume that $\left(X,\|\cdot\|_{X}\right)$ is a real Banach space, and $X^{*}$ stands for its Banach dual. We distinguish two classes of linear operators $T: L^{\Phi} \rightarrow X$ (see [OW]).

DFinition 1.1. A linear operator $T: L^{\Phi} \rightarrow X$ is said to be $\sigma$-smooth (resp. modularly continuous) if $u_{n} \stackrel{(\text { o) }}{\longrightarrow} 0$ (resp. $\left.u_{n} \stackrel{\varrho_{\Phi}}{\longrightarrow} 0\right)$ in $L^{\Phi}$ implies $\left\|T\left(u_{n}\right)\right\|_{X} \rightarrow 0$.

In Section 2, we study a relationship between $\sigma$-smooth operators, modularly continuous operators and $\left(\mathcal{T}_{\Phi}^{\wedge},\|\cdot\|_{X}\right)$-continuous linear operators $T: L^{\Phi} \rightarrow X$. It is shown that every $\sigma$-smooth linear operator $T: L^{\Phi} \rightarrow X$ factors through the inclusion map $j: L^{\Phi} \rightarrow L^{\bar{\Phi}}$, where $\bar{\Phi}$ stands for the convex minorant of $\Phi$. In Section 3, we obtain a Bochner integral representation of $\sigma$-smooth operators $T: L^{\Phi} \rightarrow X$. This extends some earlier results due to J. J. Uhl [U, Theorem 1], where $\Phi$ is supposed to be convex and $\Phi \in \Delta_{2}$.

2. Smooth operators. We first establish a relationship between different classes of linear operators $T: L^{\Phi} \rightarrow X$.

THEOREM 2.1. Let $\Phi$ be an Orlicz function. Then for a linear operator $T: L^{\Phi} \rightarrow X$ the following statements are equivalent:

(i) $T$ is modularly continuous.

(ii) $T$ is $\sigma$-smooth.

(iii) $x^{*} \circ T \in\left(L^{\Phi}\right)_{n}^{\sim}$ for all $x^{*} \in X^{*}$.

(iv) $T$ is $\left(\sigma\left(L^{\Phi}, L^{\Phi^{*}}\right), \sigma\left(X, X^{*}\right)\right)$-continuous.

(v) $T$ is $\left(\tau\left(L^{\Phi}, L^{\Phi^{*}}\right),\|\cdot\|_{X}\right)$-continuous.

(vi) $T$ is $\left(\mathcal{T}_{\bar{\Phi}}^{\wedge} \Gamma_{L^{\Phi}},\|\cdot\|_{X}\right)$-continuous.

(vii) $T$ is $\left(\mathcal{T}_{\Phi}^{\wedge},\|\cdot\|_{X}\right)$-continuous.

Proof. (i) $\Rightarrow$ (ii). Assume that $T$ is modularly continuous and let $u_{n} \stackrel{(\text { o) }}{\longrightarrow} 0$ in $L^{\Phi}$. Then by the Lebesgue dominated convergence theorem $u_{n} \stackrel{\varrho_{\Phi}}{\longrightarrow} 0$, so $\left\|T\left(u_{n}\right)\right\|_{X} \rightarrow 0$. This means that $T$ is $\sigma$-smooth.

(ii) $\Rightarrow$ (iii). Assume that $T$ is $\sigma$-smooth. Hence $x^{*} \circ T \in\left(L^{\Phi}\right)_{c}^{\sim}=\left(L^{\Phi}\right)_{n}^{\sim}$ for every $x^{*} \in X^{*}$.

(iii) $\Leftrightarrow($ iv). See $[A B$, Theorem 9.26].

(iv) $\Leftrightarrow(\mathrm{v})$. See [Wi, Corollary 11-1-3, Corollary 11-2-6].

$(\mathrm{v}) \Leftrightarrow(\mathrm{vi})$. It is obvious, because $\tau\left(L^{\Phi}, L^{\Phi^{*}}\right)=\mathcal{T}_{\bar{\Phi}} \uparrow_{L^{\Phi}}\left(\right.$ see $\left[\mathrm{N}_{4}\right.$, Theorem 2.2]).

(vi) $\Rightarrow$ (vii). Clear, because $\mathcal{T}_{\bar{\Phi}}^{\wedge} \Gamma_{L^{\Phi}} \subset \mathcal{T}_{\Phi}^{\wedge}$.

(vii) $\Rightarrow(\mathrm{i})$. It is obvious, because $u_{n} \stackrel{\varrho_{\Phi}}{\longrightarrow} 0$ in $L^{\Phi}$ implies $u_{n} \rightarrow 0$ for $\mathcal{T}_{\Phi}^{\wedge}$. 
Now, we consider the problem of extension of linear operators $T: L^{\Phi} \rightarrow X$.

Theorem 2.2. Let $\Phi$ be an Orlicz function. Assume that $T: L^{\Phi} \rightarrow X$ is a $\left(\mathcal{T}_{\Phi}^{\wedge},\|\cdot\|_{X}\right)$ continuous linear operator. Then there exists a $\left(\mathcal{T}_{\bar{\Phi}}^{\wedge},\|\cdot\|_{X}\right)$-continuous linear operator $\bar{T}: L^{\bar{\Phi}} \rightarrow X$ such that $\bar{T}(u)=T(u)$ for all $u \in L^{\Phi}$.

Proof. In view of Theorem 2.1, $T$ is $\left(\mathcal{T}_{\bar{\Phi}}^{\wedge} \uparrow_{L^{\Phi}},\|\cdot\|_{X}\right)$-continuous. Now let $u \in L^{\bar{\Phi}}$. Then there exists a sequence $\left(s_{n}\right)$ in $\mathcal{S}(\Sigma)$ such that $s_{n}(\omega) \rightarrow u(\omega) \mu$-a.e., and $\left|s_{n}(\omega)\right| \leq|u(\omega)|$ $\mu$-a.e., that is, $s_{n} \stackrel{(\mathrm{o})}{\longrightarrow} 0$ in $L^{\bar{\Phi}}$. Hence $s_{n} \rightarrow u$ for $\mathcal{T}_{\bar{\Phi}}^{\wedge}$, because $\mathcal{T}_{\bar{\Phi}}^{\wedge}$ is a Lebesgue topology on $L^{\bar{\Phi}}$. Then $\left(s_{n}\right)$ is a Cauchy sequence in $\left(L^{\Phi}, \mathcal{T}_{\bar{\Phi}}^{\wedge} \uparrow_{L^{\Phi}}\right)$, so $\left(T\left(s_{n}\right)\right)$ is a Cauchy sequence in $\left(X,\|\cdot\|_{X}\right)$. Let us put $\bar{T}(u):=\lim T\left(s_{n}\right)$ in $\left(X,\|\cdot\|_{X}\right)$. Note that if $u \in L^{\Phi}$, then $T(u)=\lim T\left(s_{n}\right)$ in $\left(X,\|\cdot\|_{X}\right)$ and $\bar{T}(u)=T(u)$.

Now we shall show that if $\left(s_{n}^{1}\right)$ and $\left(s_{n}^{2}\right)$ are sequences in $\mathcal{S}(\Sigma)$ such that $s_{n}^{1} \stackrel{(\mathrm{o})}{\longrightarrow} u$ and $s_{n}^{2} \stackrel{(\mathrm{o})}{\longrightarrow} u$ in $L^{\bar{\Phi}}$, then $\lim T\left(s_{n}^{1}\right)=\lim T\left(s_{n}^{2}\right)$ in $\left(X,\|\cdot\|_{X}\right)$. Indeed, we have $s_{n}^{1} \rightarrow u$ for $\mathcal{T}_{\bar{\Phi}}^{\wedge}$ and $s_{n}^{2} \rightarrow u$ for $\mathcal{T}_{\bar{\Phi}}^{\wedge}$, so $s_{n}^{1}-s_{n}^{2} \rightarrow 0$ for $\mathcal{T}_{\bar{\Phi}}^{\wedge} \uparrow_{L^{\Phi}}$. Hence $\left\|T\left(s_{n}^{1}\right)-T\left(s_{n}^{2}\right)\right\|_{X} \rightarrow 0$. Set $x_{1}=\lim T\left(s_{n}^{1}\right)$ and $x_{2}=\lim T\left(s_{n}^{2}\right)$ in $\left(X,\|\cdot\|_{X}\right)$. Then

$$
\left\|x_{1}-x_{2}\right\|_{X} \leq\left\|x_{1}-T\left(s_{n}^{1}\right)\right\|_{X}+\left\|T\left(s_{n}^{1}\right)-T\left(s_{n}^{2}\right)\right\|_{X}+\left\|T\left(s_{n}^{2}\right)-x_{2}\right\|_{X},
$$

and it follows that $\left\|x_{1}-x_{2}\right\|_{X}=0$, so $x_{1}=x_{2}$.

We shall now show that a linear operator $\bar{T}: L^{\bar{\Phi}} \rightarrow X$ is $\left(\mathcal{T}_{\bar{\Phi}}^{\wedge},\|\cdot\|_{X}\right)$-continuous. Indeed, let $B_{\mathcal{T}_{\bar{\Phi}}}$ stand for the local base at 0 for $\mathcal{T}_{\bar{\Phi}}^{\wedge}$, and let $\varepsilon>0$ be given. Since $T$ is $\left(\mathcal{T}_{\bar{\Phi}}^{\wedge} \uparrow_{L^{\Phi}},\|\cdot\|_{X}\right)$-continuous, there exists $W \in B_{\mathcal{T}_{\bar{\Phi}}}$ such that $T\left(L^{\Phi} \cap W\right) \subset B_{X}(\varepsilon)$ $\left(=\left\{x \in X:\|x\|_{X} \leq \varepsilon\right\}\right)$. It is enough to show that $\bar{T}(W) \subset B_{X}(\varepsilon)$. In fact, let $w \in W$. Then there exists a sequence $\left(s_{n}\right)$ in $\mathcal{S}(\Sigma)$ such that $s_{n} \rightarrow w$ for $\mathcal{T}_{\bar{\Phi}}$. Hence there exists $n_{0} \in \mathbb{N}$ such that $s_{n} \in L^{\Phi} \cap W$ for all $n \geq n_{0}$; so $T\left(s_{n}\right) \in B_{X}(\varepsilon)$ for $n \geq n_{0}$. It follows that $\bar{T}(w) \in B_{X}(\varepsilon)$, as desired.

As a consequence of Theorem 2.1 and Theorem 2.2 we obtain the following factorization of $\sigma$-smooth operators $T: L^{\Phi} \rightarrow X$.

COROLlary 2.3. Let $\Phi$ be an Orlicz function and let $T: L^{\Phi} \rightarrow X$ be a $\sigma$-smooth linear operator. Then $T$ may be factorized: $T=\bar{T} \circ j$, where $j: L^{\Phi} \rightarrow L^{\bar{\Phi}}$ is the inclusion map and $\bar{T}: L^{\bar{\Phi}} \rightarrow X$ is a $\sigma$-smooth linear operator.

3. Integral representation of smooth operators. In this section we obtain a Bochner integral representation of $\sigma$-smooth linear operators $T: L^{\Phi} \rightarrow X$, where $\Phi$ is an Orlicz function (not necessarily convex) and $X$ has the Radon-Nikodym Property. We extend some earlier results due to J. J. Uhl (see [U, Theorem 1]), where $\Phi$ is supposed to be convex and $\Phi \in \Delta_{2}$. The problem of Bochner integral representation of linear operators $T: L^{p} \rightarrow X(p>1)$ has been studied in [DU, Theorem 3.4.8], [D $]$, [ $\left.\mathrm{D}_{2}\right],\left[\mathrm{D}_{3}\right]$.

For terminology concerning vector measures and Banach-space valued function spaces we refer to [DU, Chap. 3.1], [L]. Denote by $L^{0}(X)$ the set of $\mu$-equivalence classes of all strongly $\Sigma$-measurable functions $g: \Omega \rightarrow X$. For $g: \Omega \rightarrow X$ let us put $\widetilde{g}(\omega)=\|g(\omega)\|_{X}$ for $\omega \in \Omega$. For an Orlicz function $\Phi$ the Orlicz-Bochner space $L^{\Phi}(X)$ is defined by

$$
L^{\Phi}(X)=\left\{g \in L^{0}(X): \widetilde{g} \in L^{\Phi}\right\} .
$$


A linear operator $T: L^{\Phi} \rightarrow X$ is said to be regular if there exists $0 \leq v \in L^{\Phi^{*}}$ such that $\|T(u)\|_{X} \leq \varphi_{v}(|u|)=\int_{\Omega}|u(\omega)| v(\omega) d \mu$ for all $u \in L^{\Phi}$ (see [Bu, Def. 1.2]).

From now on we will assume that $(\Omega, \Sigma, \mu)$ is a finite atomless measure space. Recall that a Banach space $X$ has the Radon-Nikodym property (with respect to $\mu$ ) (briefly $X \in R N P(\mu))$ if for each $\mu$-continuous vector measure $m: \Sigma \rightarrow X$ of bounded variation (i.e., $|m|(\Omega)<\infty)$ there exists $g \in L^{1}(X)$ such that

$$
m(A)=\int_{A} g(\omega) d \mu \quad \text { for all } A \in \Sigma .
$$

Then $|m|(A)=\int_{A}\|g(\omega)\|_{X} d \mu$ for all $A \in \Sigma$. Motivated by the variation $|m|(\Omega)$ and following $\left[\mathrm{D}_{1}\right],\left[\mathrm{D}_{3}\right]$ we can define a norm functional of operators $T: L^{\Phi} \rightarrow X$ by

$$
\|T\|_{\Phi}:=\sup \left\{\sum_{i=1}^{n}\left\|\alpha_{i} T\left(1_{A_{i}}\right)\right\|_{X}: s=\sum_{i=1}^{n} \alpha_{i} 1_{A_{i}} \in \mathcal{S}(\Sigma), \varrho_{\Phi}(s) \leq 1\right\} .
$$

Now we are in a position to state our main result.

THEOREM 3.1. Let $\Phi$ be an Orlicz function and let $X \in R N P(\mu)$. Then for a linear operator $T: L^{\Phi} \rightarrow X$ the following statements are equivalent:

(i) $\|T\|_{\Phi}<\infty$ and $T$ is modularly continuous.

(ii) $\|T\|_{\Phi}<\infty$ and $T$ is $\sigma$-smooth.

(iii) $\|T\|_{\Phi}<\infty$ and $T$ is $\left(\tau\left(L^{\Phi}, L^{\Phi^{*}}\right),\|\cdot\|_{X}\right)$-continuous.

(iv) $\|T\|_{\Phi}<\infty$ and $T$ is $\left(\mathcal{T}_{\Phi}^{\wedge},\|\cdot\|_{X}\right)$-continuous.

(v) There exists $g \in L^{\Phi^{*}}(X)$ such that

$$
T(u)=T_{g}(u)=\int_{\Omega} u(\omega) g(\omega) d \mu \quad \text { for all } u \in L^{\Phi}
$$

and

$$
\left\|T_{g}\right\|_{\Phi}=\left\|\varphi_{\widetilde{g}}\right\|_{\Phi}=\sup \left\{\left|\int_{\Omega} u(\omega) \widetilde{g}(\omega) d \mu\right|: u \in L^{\Phi}, \varrho_{\Phi}(u) \leq 1\right\} .
$$

In particular, if $\Phi$ is a convex Orlicz function, then

$$
\left\|T_{g}\right\|_{\Phi}=\|\widetilde{g}\|_{\Phi^{*}}^{0}=\|g\|_{L^{\Phi^{*}}(X)}^{0} .
$$

(vi) $T$ is regular.

Proof. (i) $\Leftrightarrow($ ii $) \Leftrightarrow($ iii $) \Leftrightarrow$ (iv) follow from Theorem 2.1.

(i) $\Rightarrow$ (v). Assume that $\|T\|_{\Phi}<\infty$ and $T$ is modular continuous.

Define a vector measure $m_{T}: \Sigma \rightarrow X$ by $m_{T}(A)=T\left(1_{A}\right)$ for $A \in \Sigma$. We shall now show that $m_{T}$ is $\mu$-continuous. Indeed, let $\mu\left(A_{n}\right) \rightarrow 0$ with $A_{n} \in \Sigma$. Then

$$
\varrho_{\Phi}\left(1_{A_{n}}\right)=\int_{\Omega} \Phi\left(1_{A_{n}}(\omega)\right) d \mu=\Phi(1) \mu\left(A_{n}\right) \rightarrow 0,
$$

so

$$
\left\|m_{T}\left(A_{n}\right)\right\|_{X}=\left\|T\left(1_{A_{n}}\right)\right\|_{X} \rightarrow 0 .
$$

It follows that $m_{T}$ is countably additive and $\mu$-continuous. Now, choose $\alpha>0$ such that $\varrho_{\Phi}\left(\alpha 1_{\Omega}\right) \leq 1$. For any finite $\Sigma$-partition $\left\{A_{i}: 1 \leq i \leq n\right\}$ of $\Omega$ we have $\alpha 1_{\Omega}=\sum_{i=1}^{n} \alpha 1_{A_{i}}$, 
so

$$
\alpha \sum_{i=1}^{n}\left\|m_{T}\left(A_{i}\right)\right\|_{X}=\sum_{i=1}^{n}\left\|\alpha T\left(1_{A_{i}}\right)\right\|_{X} \leq\|T\|_{\Phi} .
$$

Hence $\left|m_{T}\right|(\Omega)<\infty$, and since $X \in R N P(\mu)$ there exists $g \in L^{1}(X)$ such that

$$
m_{T}(A)=\int_{A} g(\omega) d \mu \quad \text { and } \quad\left|m_{T}\right|(A)=\int_{A}\|g(\omega)\|_{X} d \mu \quad \text { for } A \in \Sigma .
$$

Then for $s=\sum_{i=1}^{n} \alpha_{i} 1_{A_{i}} \in \mathcal{S}(\Sigma)$ we have

$$
\begin{aligned}
T(s) & =\sum_{i=1}^{n} \alpha_{i} T\left(1_{A_{i}}\right)=\sum_{i=1}^{n} \alpha_{i} m_{T}\left(A_{i}\right) \\
& =\sum_{i=1}^{n} \alpha_{i} \int_{A_{i}} g(\omega) d \mu=\int_{\Omega} s(\omega) g(\omega) d \mu .
\end{aligned}
$$

We now show that for $s=\sum_{i=1}^{n} \alpha_{i} 1_{A_{i}} \in \mathcal{S}(\Sigma)$ with $\varrho_{\Phi}(s) \leq 1$ we have

$$
\sum_{i=1}^{n}\left|\alpha_{i}\right| \int_{A_{i}}\|g(\omega)\|_{X} d \mu=\sum_{i=1}^{n}\left|\alpha_{i}\right|\left|m_{T}\right|\left(A_{i}\right) \leq\|T\|_{\Phi}
$$

Indeed, let $\varepsilon>0$ be given. Then for each $1 \leq i \leq n$ there exists a $\Sigma$-partition $\left(A_{i, j}\right)_{j=1}^{k_{i}}$ of $A_{i}$ such that

$$
\left|m_{T}\right|\left(A_{i}\right) \leq \sum_{j=1}^{k_{i}}\left\|m_{T}\left(A_{i, j}\right)\right\|_{X}+\frac{\varepsilon}{n\left|\alpha_{i}\right|}=\sum_{j=1}^{k_{i}}\left\|T\left(1_{A_{i, j}}\right)\right\|_{X}+\frac{\varepsilon}{n\left|\alpha_{i}\right|} .
$$

Hence

$$
\sum_{i=1}^{n}\left|\alpha_{i}\right|\left|m_{T}\right|\left(A_{i}\right) \leq \sum_{i=1}^{n}\left(\sum_{j=1}^{k_{i}}\left\|\alpha_{i} T\left(1_{A_{i, j}}\right)\right\|_{X}\right)+\varepsilon \leq\|T\|_{\Phi}+\varepsilon
$$

because

$$
\sum_{i=1}^{n}\left(\sum_{j=1}^{k_{i}} \alpha_{i} 1_{A_{i, j}}\right)=\sum_{i=1}^{n} \alpha_{i} 1_{A_{i}}
$$

Then

$$
\begin{aligned}
\sum_{i=1}^{n}\left\|\alpha_{i} T\left(1_{A_{i}}\right)\right\|_{X} & =\sum_{i=1}^{n}\left|\alpha_{i}\right|\left\|m_{T}\left(A_{i}\right)\right\|_{X} \leq \sum_{i=1}^{n}\left|\alpha_{i}\right|\left|m_{T}\right|\left(A_{i}\right) \\
& =\sum_{i=1}^{n}\left|\alpha_{i}\right| \int_{A_{i}}\|g(\omega)\|_{X} d \mu=\int_{\Omega}\left(\sum_{i=1}^{n}\left|\alpha_{i}\right| 1_{A_{i}}(\omega)\right)\|g(\omega)\|_{X} d \mu \\
& =\int_{\Omega}|s(\omega)| \widetilde{g}(\omega) d \mu \leq\|T\|_{\Phi} .
\end{aligned}
$$

Taking suprema on the left, we get

$$
\|T\|_{\Phi}=\sup \left\{\int_{\Omega}|s(\omega)| \widetilde{g}(\omega) d \mu: s \in \mathcal{S}(\Sigma), \varrho_{\Phi}(s) \leq 1\right\} .
$$

Now we are ready to show $u \widetilde{g} \in L^{1}$ for every $u \in L^{\Phi}$, i.e., $\widetilde{g} \in\left(L^{\Phi}\right)^{\prime}=L^{\Phi^{*}}$. Indeed, let $u \in L^{\Phi}$. Then there exists a sequence $\left(s_{n}\right)$ in $\mathcal{S}(\Sigma)$ such that $0 \leq s_{n}(\omega) \uparrow|u(\omega)|$ for 
$\omega \in \Omega$ (see [KA, Corollary I.6]). Choose $\alpha>0$ such that $\varrho_{\Phi}(\alpha u) \leq 1$. Then by Fatou's lemma and (3.2) we get

$$
\int_{\Omega} \alpha|u(\omega)| \widetilde{g}(\omega) d \mu \leq \sup _{n} \int_{\Omega} \alpha s_{n}(\omega) \widetilde{g}(\omega) d \mu \leq\|T\|_{\Phi},
$$

and this means that $\widetilde{g} \in\left(L^{\Phi}\right)^{\prime}=L^{\Phi^{*}}$ and $u g \in L^{1}(X)$. Thus we can define a linear operator $T_{g}: L^{\Phi} \rightarrow X$ by

$$
T_{g}(u)=\int_{\Omega} u(\omega) g(\omega) d \mu \quad \text { for } u \in L^{\Phi} .
$$

We shall now show that $T_{g}(u)=T(u)$ for $u \in L^{\Phi}$. Indeed, let $u \in L^{\Phi}$ and choose $\alpha>0$ such that $\varrho_{\Phi}(2 \alpha u)<\infty$. Then there exists a sequence $\left(s_{n}\right)$ in $\mathcal{S}(\Sigma)$ such that $s_{n}(\omega) \rightarrow u(\omega) \mu$-a.e. and $\left|s_{n}(\omega)\right| \leq|u(\omega)| \mu$-a.e. ([KA, Corollary I.6]). By the dominated convergence theorem $\varrho_{\Phi}\left(\alpha\left(s_{n}-u\right)\right) \rightarrow 0$, and since $T$ is modularly continuous, we get $\left\|T\left(s_{n}\right)-T(u)\right\|_{X} \rightarrow 0$.

On the other hand, $s_{n}(\omega) \widetilde{g}(\omega) \rightarrow u(\omega) \widetilde{g}(\omega) \mu$-a.e. and $\left|s_{n}(\omega)\right| \widetilde{g}(\omega) \leq|u(\omega)| \widetilde{g}(\omega) \mu$-a.e., where $u \widetilde{g} \in L^{1}$. Using (3.1) we get

$$
\begin{aligned}
\left\|T\left(s_{n}\right)-T_{g}(u)\right\|_{X} & =\left\|\int_{\Omega} s_{n}(\omega) g(\omega) d \mu-\int_{\Omega} u(\omega) g(\omega) d \mu\right\|_{X} \\
& \leq \int_{\Omega}\left|s_{n}(\omega)-u(\omega)\right| \widetilde{g}(\omega) d \mu \underset{n}{\longrightarrow} 0 .
\end{aligned}
$$

It follows that

$$
T(u)=T_{g}(u)=\int_{\Omega} u(\omega) g(\omega) d \mu \quad \text { for } u \in L^{\Phi} .
$$

Now assume that $\Phi$ is a convex Orlicz function. Then $\varrho_{\Phi}(u) \leq 1$ if and only if $\|u\|_{\Phi} \leq 1$ and it follows that $\left\|T_{g}\right\|_{\Phi}=\|\widetilde{g}\|_{\Phi}^{0}=\|g\|_{L^{\Phi^{*}}(X)}^{0}$.

$(\mathrm{v}) \Rightarrow(\mathrm{vi})$. Assume that there exists $g \in L^{\Phi^{*}}(X)$ such that

$$
T(u)=T_{g}(u)=\int_{\Omega} u(\omega) g(\omega) d \mu \quad \text { for all } u \in L^{\Phi} .
$$

Then for $u \in L^{\Phi}$ we have

$$
\|T(u)\|_{X} \leq \int_{\Omega}|u(\omega)|\|g(\omega)\|_{X} d \mu=\varphi_{\widetilde{g}}(|u|),
$$

where $\widetilde{g} \in L^{\Phi^{*}}$, i.e., $T$ is regular.

(vi) $\Rightarrow$ (ii). Assume that $T$ is regular, i.e., there exists $0 \leq v \in L^{\Phi^{*}}$ such that

$$
\|T(u)\|_{X} \leq \int_{\Omega}|u(\omega)| v(\omega) d \mu=\varphi_{v}(|u|) \quad \text { for all } u \in L^{\Phi} .
$$

Let $s=\sum_{i=1}^{n} \alpha_{i} 1_{A_{i}} \in \mathcal{S}(\Sigma)$ with $\varrho_{\Phi}(s) \leq 1$. Then using (3.1) we get

$$
\begin{aligned}
\sum_{i=1}^{n}\left\|\alpha_{i} T\left(1_{A_{i}}\right)\right\|_{X} & =\sum_{i=1}^{n}\left|\alpha_{i}\right|\left\|T\left(1_{A_{i}}\right)\right\|_{X} \leq \sum_{i=1}^{n}\left|\alpha_{i}\right| \int_{\Omega} 1_{A_{i}}(\omega) v(\omega) d \mu \\
& =\int_{\Omega}\left(\sum_{i=1}^{n}\left|\alpha_{i}\right|\left(1_{A_{i}}\right)(\omega)\right) v(\omega) d \mu=\varphi_{v}(|s|) \\
& \leq\left\|\varphi_{\tilde{g}}\right\|_{\Phi}\left(\varrho_{\Phi}(s)+1\right) \leq 2\left\|\varphi_{\tilde{g}}\right\|_{\Phi} .
\end{aligned}
$$


Hence $\left\|T_{g}\right\|_{\Phi} \leq 2\left\|\varphi_{\tilde{g}}\right\|_{\Phi}$. Now assume that $u_{n} \stackrel{(\mathrm{o})}{\longrightarrow} 0$ in $L^{\Phi}$. Since $\varphi_{v} \in\left(L^{\Phi}\right)_{n}^{\sim}$, we obtain that $\left\|T\left(u_{n}\right)\right\|_{X} \rightarrow 0$, i.e., $T$ is $\sigma$-smooth.

\section{References}

[AB] C. D. Aliprantis and O. Burkinshaw, Positive Operators, Academic Press, Orlando, 1985.

[Bu] A. V. Bukhvalov, On an analytic representation of linear operators using vector-valued measurable function, Izv. Vyssh. Ucheb. Zaved. 7 (1977), 21-31.

[C] J. Chaney, Banach lattices of compact maps, Math. Z. 129 (1972) 1-19.

$\left[D_{1}\right] \quad$ N. Dinculeanu, Vector Measures, Pergamon Press, New York, 1967.

$\left[\mathrm{D}_{2}\right] \quad \mathrm{N}$. Dinculeanu, Integral representation of linear operators, I, II, Stud. Cerc. Mat. (1966), 349-385, 483-536.

$\left[D_{3}\right] \quad$ N. Dinculeanu, Linear operators on $L^{p}$-spaces, in: Vector and Operator Valued Measures and Applications (Proc. Sympos., Utah 1972), Academic Press New York, 1973, 109-124.

[DU] J. Diestel and J. J. Uhl, Vector Measures, Math. Surveys 15, Amer. Math. Soc., 1977, Providence, RI.

[D] N. Dunford, Integration and linear operators, Trans. Amer. Math. Soc. 40 (1936) 474494.

[DP] N. Dunford and J. Pettis, Linear operations on summable functions, Trans. Amer. Math. Soc. 47 (1940), 323-392.

[DS] N. Dunford and J. Schwartz, Linear Operators, Part I, General Theory, Interscience Publ. Inc., New York, 1958.

[G] I. Gelfand, Abstrakte Funktionen und Lineare Operatoren, Mat. Sbornik, N.S., 4 (46) (1938), 235-238.

[K] N. Kalton, Compact and strictly singular operators on Orlicz spaces, Israel J. Math., 26 (1977), 126-136.

[KA] L. V. Kantorovich and A. V. Akilov, Functional Analysis, 3rd ed., Nauka, Moscow, 1984 (in Russian).

[L] P.-K. Lin, Köthe-Bochner Function Spaces, Birkhaüser, Boston, 2003.

[MW] L. Maligranda and W. Wnuk, Landau type theorem for Orlicz spaces, Math. Z. 208 (1991), 57-64.

[MaO] S. Mazur and W. Orlicz, On some classes of linear spaces, Studia Math. 17 (1958), 97-119.

[M] J. Musielak, Orlicz Spaces and Modular Spaces, Lecture Notes in Math. 1034, Springer Verlag, Berlin, 1983.

$\left[\mathrm{MO}_{1}\right] \quad$ J. Musielak and W. Orlicz, On modular spaces, Studia Math. 18 (1959), 49-65.

$\left[\mathrm{MO}_{2}\right]$ J. Musielak and W. Orlicz, Some remarks on modular spaces, Bull. Acad. Polon. Sci. Sér. Sci. Math. Astronom. Phys. 7 (1959), 661-668.

$\left[\mathrm{N}_{1}\right] \quad$ M. Nowak, On modular topology on Orlicz spaces, Bull. Pol. Acad. Sci. Math. 36 (1988), $553-562$.

$\left[\mathrm{N}_{2}\right] \quad$ M. Nowak, Orlicz lattices with modular topology I, Comment. Math. Univ. Carolinae 30 (1989), 261-270.

[N $\left.\mathrm{N}_{3}\right]$ M. Nowak, Order continuous linear functionals on non-locally convex Orlicz spaces, Comment. Math. Univ. Carolinae 33 (1992), 465-475. 
$\left[\mathrm{N}_{4}\right] \quad$ M. Nowak, On the strongest locally convex Lebesgue topology on Orlicz spaces, Results Math. 33 (1998), 134-138.

[O] W. Orlicz, On integral representability of linear functionals over the space of $\varphi$ integrable functions, Bull. Acad. Polon. Sci. Sér. Sci. Math. Astronom. Phys. 7 (1959), 661-668.

[OW] W. Orlicz and W. Wnuk, Absolutely continuous and modularly continuous operators defined on spaces of measurable functions, Ricerche di Matematica 60 (1991), 243-258.

[P] D. Pallaschke, The compact endomorphism of the metric linear space $\mathcal{L}_{\Phi}$, Studia Math. 47 (1973), 123-133.

[Ph] R. S. Phillips, On linear transformations, Trans. Amer. Math. Soc. 48 (1940), 516-541.

[RR] M. M. Rao and Z. D. Ren, Theory of Orlicz Spaces, Marcel Dekker, New York, 1991.

$\left[\mathrm{T}_{1}\right] \quad \mathrm{Ph}$. Turpin, Opérateurs linéaires entre espaces d'Orlicz non localement convexes, Studia Math. 46 (1973), 153-165.

$\left[\mathrm{T}_{2}\right] \quad \mathrm{Ph}$. Turpin, Convexités dans les espaces vectoriels topologiques généraux, Dissert. Math. 131 (1976).

[U] J. J. Uhl, On a class of operators on Orlicz spaces, Studia Math. 40 (1971), 17-22.

[Wi] A. Wilansky, Modern Methods in Topological Vector Spaces, McGraw-Hill, 1978.

[W] T. K. Wong, On a class of absolutely p-summing operators, Studia Math. 39 (1971), 181-189.

[Z] A. C. Zaanen, Linear Analysis, North-Holland, Amsterdam, 1953. 
